\title{
Unconventional solution-phase epitaxial growth of organic-inorganic hybrid perovskite nanocrystals on metal sulfide nanosheets
}

\author{
Zhipeng Zhang ${ }^{1}$, Fangfang Sun ${ }^{1}$, Zhaohua Zhu ${ }^{1}$, Jie Dai ${ }^{1}$ Kai Gao ${ }^{1}$, Qi Wei ${ }^{1}$, Xiaotong Shi ${ }^{1}$, \\ Qian Sun ${ }^{1}$, Yan Yan ${ }^{1}, \mathrm{Hai}_{\mathrm{Li}^{1}}{ }^{1}$, Haidong Yu${ }^{1}$, Guichuan Xing ${ }^{1,2^{*}}$, Xiao Huang ${ }^{1^{*}}$ and Wei Huang ${ }^{1,3^{*}}$
}

\begin{abstract}
Epitaxial heterostructures based on organicinorganic hybrid perovskites and two-dimensional materials hold great promises in optoelectronics, but they have been prepared only via solid-state methods that restricted their practical applications. Herein, we report cubic-phased $\mathrm{MAPbBr}_{3}\left(\mathrm{MA}=\mathrm{CH}_{3} \mathrm{NH}_{3}{ }^{+}\right)$nanocrystals were epitaxially deposited on trigonal/hexagonal-phased $\mathrm{MoS}_{2}$ nanosheets in solution by facilely tuning the solvation conditions. In spite of the mismatched lattice symmetry between the square $\mathrm{MAPbBr}_{3}(001)$ overlayer and the hexagonal $\mathrm{MoS}_{2}(001)$ substrate, two different aligning directions with lattice mismatch of as small as $1 \%$ were observed based on the domainmatching epitaxy. This was realized most likely due to the flexible nature and absence of surface dangling bonds of $\mathrm{MoS}_{2}$ nanosheets. The formation of the epitaxial interface affords an effective energy transfer from $\mathrm{MAPbBr}_{3}$ to $\mathrm{MoS}_{2}$, and as a result, paper-based photodetectors facilely fabricated from these solution-dispersible heterostructures showed better performance compared to those based on $\mathrm{MoS}_{2}$ or $\mathrm{MAPbBr}_{3}$ alone. In addition to the improved energy transfer and light adsorption, the use of $\mathrm{MoS}_{2}$ nanosheets provided flexible and continuous substrates to connect the otherwise discrete $\mathrm{MAPbBr}_{3}$ nanocrystals and achieved the better film forming ability. Our work suggests that the scalable preparation of heterostructures based on organic-inorganic hybrid perovskites and 2D materials via solution-phase epitaxy may bring about more opportunities for expanding their optoelectronic applications.
\end{abstract}

Keywords: organic-inorganic hybrid perovskite, transition metal chalcogenide, epitaxial growth, paper-based photodetector

\section{INTRODUCTION}

In the past decade, intensive research effort has been devoted to two-dimensional (2D) layered materials [1-3]. Among the various $2 \mathrm{D}$ materials, transition metal dichalcogenide (TMDs) nanosheets have drawn particular attention due to their promises in electrocatalysis [4], electronics [5], optoelectronics [6] and so on. However, in terms of light-conversion/emitting properties, semiconducting TMDs generally exhibited low quantum efficiencies partly due to their large specific surface area accompanied with a high density of surface defects that can act as recombination centers [7]. Besides, TMD nanosheets exhibited limited light-absorbing ability because of their atomic-scale thicknesses. For instance, a singlelayer $\mathrm{MoS}_{2}$ absorbed only $10 \%$ of incident photons at its excitonic resonances (i.e., 615 and $660 \mathrm{~nm}$ ) [8]. Much effort has thus been made to create TMD-based heterostructures like $\mathrm{MoS}_{2}$ /graphene [9] and $\mathrm{MoS}_{2} / \mathrm{WS}_{2}$, [10] to improve the efficiency of charge separation/transport. Enhanced light absorption has also been achieved by hybridizing TMDs with noble metal nanoparticles [11], organic dyes [12], silicon (Si) [13], carbon nanotubes [14] and so on.

Organic-inorganic hybrid perovskites (e.g., $\mathrm{CH}_{3} \mathrm{NH}_{3}$ $\mathrm{PbI}_{3}$ ) have recently emerged as a class of promising optoelectronic materials [15-17]. Solar cells based on them have achieved remarkable power conversion efficiencies exceeding 20\% [18-20]. The excellent performance has been attributed to a combination of several attractive features such as a high and balanced carrier mobility [21], long carrier diffusion length [22] and large light absorp-

\footnotetext{
${ }^{1}$ Key Laboratory of Flexible Electronics (KLOFE) \& Institute of Advanced Materials (IAM), Jiangsu National Synergistic Innovation Center for Advanced Materials (SICAM), Nanjing Tech University (NanjingTech), Nanjing 211816, China

${ }^{2}$ Institute of Applied Physics and Materials Engineering, University of Macau, Macau SAR 999078, China

${ }^{3}$ Shaanxi Institute of Flexible Electronics (SIFE), Northwestern Polytechnical University (NPU), Xi'an 710072, China

* Corresponding authors (emails: iamgcxing@njtech.edu.cn (Xing G); iamxhuang@njtech.edu.cn (Huang X); iamwhuang@njtech.edu.cn (Huang W))
} 
tion coefficient in the UV-vis range [23]. Recently, organic-inorganic hybrid perovskites in the form of colloidal nanocrystals (NCs) have been reported [24,25], and compared to their bulk counterpart, they present a number of advantages such as a high photoluminescence (PL) quantum yield [26], narrow-band emission [27] and shape-dependent PL [28]. These merits make them especially attractive for applications such as solutionprocessed photodetectors [29], light-emitting diodes [30] and solar cells [31]. Unfortunately, colloidal NCs with small lateral sizes suffer from poor film-forming ability, restricting their use in applications where continuous films for charge transporting are required. Recently, TMDs and organic-inorganic hybrid perovskites have been combined into heterostructures, with the aim to marrying their good electronic and optical properties [32-37]. For example, photoconductors based on $\mathrm{WSe}_{2} /$ $\mathrm{CH}_{3} \mathrm{NH}_{3} \mathrm{PbI}_{3}$ [34] and $\mathrm{WS}_{2} / \mathrm{CH}_{3} \mathrm{NH}_{3} \mathrm{PbI}_{3}$ [35] demonstrated superior photo-responsive performance compared to single-component devices. However, such heterojunctions have been realized mostly via solid-state methods typically involving chemical vapor deposition (CVD), [35] mechanical exfoliation [36] and/or dry transfer [33], which are difficult to scale-up for practical applications. Direct growth of perovskite crystals on dispersible 2D materials in solution is expected to enable the scalable production of solution-processible heterostructures, but has not been realized, because a non-polar solvent is usually needed for the precipitation of perovskite crystals $[38,39]$ which is incompatible with most solvation conditions for $2 \mathrm{D}$ materials.

In this contribution, we demonstrated a facile wetchemical method to directly grow organic-inorganic hybrid perovskite $\left(\mathrm{MAPbBr}_{3}, \mathrm{MA}=\mathrm{CH}_{3} \mathrm{NH}_{3}{ }^{+}\right) \mathrm{NCs}$ on surfaces of dispersible $\mathrm{MoS}_{2}$ nanosheets. Two unusual symmetry-mismatched epitaxial relationships were observed, thanks to the use of flexible and dangling-bondfree $\mathrm{MoS}_{2}$ nanosheets. We further applied the $\mathrm{MAPbBr}_{3} /$ $\mathrm{MoS}_{2}$ epitaxial heterostructures in paper-based photodetector with the much improved performance compared to their single components.

\section{EXPERIMENTAL SECTION}

Materials: $\mathrm{PbBr}_{2}$ (lead (II) bromide, 99.999\%, Sigma-Aldrich, Germany), $\mathrm{CH}_{3} \mathrm{NH}_{3} \mathrm{Br}$ (methylammonium bromide, $\geq 99.5 \%$, Xi'an Polymer Light Technology Co., Ltd., China), oleic acid (OA, $>85.0 \%$, TCI, Japan), $n$-octylamine (OLA, $>98.0 \%$, TCI, Japan), molybdenum disulfide $\left(\mathrm{MoS}_{2},<2 \mu \mathrm{m}, 99 \%\right.$, Sigma-Aldrich, Germany), acetylene black (Li Zhiyuan Co., Ltd., China), poly(vinylidene fluoride) (PVDF, Shanghai Chemical Reagent Co., Ltd., China), N,N-dimethylformamide (DMF, analytical reagent, Shanghai Chemical Reagent Co., Ltd., China), toluene (analytical reagent, Shanghai Chemical Reagent Co., Ltd., China), acetone (analytical reagent, Shanghai Chemical Reagent Co., Ltd., China) and $\mathrm{N}$-methyl-2pyrrolidone (NMP, analytical reagent, Shanghai Chemical Reagent Co., Ltd., China) were used as received without further purification. The deionized (DI) water was purified using Milli-Q3 System (Millipore, France).

Preparation of $\mathrm{MoS}_{2}$ nanosheets: $\mathrm{MoS}_{2}$ nanosheets were prepared by a previously reported electrochemical lithium intercalation and exfoliation method [40]. Layered bulk $\mathrm{MoS}_{2}$ crystals were mixed with acetylene black and PVDF binder dispersed in NMP. This mixture was then uniformly coated on a copper foil and dried under vacuum overnight. The intercalation was conducted in a battery test cell using a $\mathrm{Li}$ foil as the anode, and the $\mathrm{MoS}_{2}$ crystals coated on copper foil as the cathode. Assembly of the test cells was conducted in an Ar-filled glove box. The electrochemical Li intercalation was accomplished in a Land battery test system at a constant current density of $0.025 \mathrm{~mA}$. After the discharge process, the Li-intercalated $\mathrm{MoS}_{2}$ was washed with acetone, followed by exfoliation under ultrasonication in DI water in a closed vial to give a suspension of isolated nanosheets. The product was centrifuged at $3,000 \mathrm{rpm}$ for $20 \mathrm{~min}$ to remove the precipitate containing thicker crystals and the supernatant was washed three times with DMF before further use.

Preparation of $\mathrm{MAPbBr}_{3} / \mathrm{MoS}_{2}$ heterostructure: $0.1 \mathrm{~mL}$ of the aforementioned $\mathrm{MoS}_{2}$ suspension in DMF $\left.(\sim 1 \mathrm{mg} \mathrm{mL})^{-1}\right)$ was added dropwise into $3 \mathrm{~mL}$ toluene under ultrasonication to form a homogeneous suspension denoted as solution A. Then $0.16 \mathrm{mmol} \mathrm{CH}_{3} \mathrm{NH}_{3} \mathrm{Br}$ and $0.2 \mathrm{mmol} \mathrm{PbBr}_{2}$ was dissolved in $5 \mathrm{~mL}$ of DMF together with $20 \mu \mathrm{L}$ of OLA and $0.5 \mathrm{~mL}$ of OA to form a precursor solution B. After that, $20 \mu \mathrm{L}$ of solution B was added dropwise into solution A with mild shake for $10 \mathrm{~s}$. Upon mixing, a brownish green solution was formed. After centrifugation at $12,000 \mathrm{rpm}$ for $10 \mathrm{~min}$, the precipitates were collected and washed three times with toluene before further characterizations.

Fabrication of paper-based photodetector: a piece of commonly used writing paper (Deli, China) was used as the substrate. Two graphite electrodes with a $0.5 \mathrm{~mm}$ spacing were then drawn onto the paper using a $12 \mathrm{~B}$ pencil (Fig. S7). After that, $3 \mu \mathrm{L}$ of a concentrated dispersion of the $\mathrm{MAPbBr}_{3} / \mathrm{MoS}_{2}$ heterostructures $\left(\sim 5.0 \mathrm{mg} \mathrm{mL}^{-1}\right)$ was drop-casted between the electrodes.

Photoresponse measurements: the current-voltage 
$(I-V)$ and the current-time $(I-t)$ curves of the photodetectors were measured on a 4200 semiconductor characterization system (Keithley, USA) in air at room temperature. A $405 \mathrm{~nm}$ laser was used for all the measurements. The actual power intensity was measured by a LP1 power meter (Sanwa Electric Instrument Co., Ltd., Japan). The responsivity $(R)$ was calculated as $R=\Delta I /$ $(P \cdot S)$, where $\Delta I$ is the difference between the photocurrent and the dark current, $P$ is the incident power density, and $S$ is the effectively illuminated area.

Characterizations: the morphological and structural characterizations of the products were conducted by transmission electron microscope (TEM, HT7700, Hitachi, Japan), high resolution transmission electron microscope (HRTEM, JEOL 2100F, Japan) and X-ray diffraction (XRD, SmartLab Rigaku, Japan, with $\mathrm{Cu} \mathrm{K}_{\alpha}$ radiation at $\lambda=1.54 \AA$ ). For the $\mathrm{X}$-ray photoelectron spectroscopy (XPS, PHI 5000 VersaProbe, Japan) measurements, the binding energies were corrected for specimen charging effects using the $\mathrm{C} 1 \mathrm{~s}$ level at $284.6 \mathrm{eV}$ as the reference. UV-vis absorption spectra of the materials were measured on a UV-vis spectrophotometer (UV1750, Shimadzu, Japan). PL spectra were taken using a F4600 fluorescence spectrometer (Hitachi, Japan). The time-resolved photoluminescence (TRPL) spectroscopy spectra were acquired using a FLSP920 fluorescence spectrophotometer (Edinburgh Instruments, England).

\section{RESULTS AND DISCUSSION}

Typically, $\mathrm{MoS}_{2}$ nanosheets were prepared by electrochemical lithium intercalation and exfoliation based on a previous report (see the experimental section for the detailed procedure) [40]. Based on TEM and atomic force microscopy (AFM) measurements (Fig. S1), the as-prepared $\mathrm{MoS}_{2}$ nanosheets were $200-500 \mathrm{~nm}$ in lateral size and $1.3-3.0 \mathrm{~nm}$ in thickness, suggesting that they are single- to few-layer thick [41]. The subsequent preparation of $\mathrm{MAPbBr}_{3} / \mathrm{MoS}_{2}$ heterostructures is schematically illustrated in Fig. 1a. First, the as-exfoliated $\mathrm{MoS}_{2}$ nanosheets were redispersed in a mixed solvent composing of DMF and toluene with a volumetric ratio of $V_{\mathrm{DMF}}$ : $V_{\text {toluene }}=1: 30$. To this $\mathrm{MoS}_{2}$ suspension, a mixture of $\mathrm{CH}_{3}$ $\mathrm{NH}_{3} \mathrm{Br}, \mathrm{PbBr}_{2}$, OA, and OLA in DMF was added dropwise with mild shake. Upon mixing, the solution color changed from brown to brownish green, indicating the formation of perovskite NCs. The scanning transmission electron microscopy (STEM) image in Fig. 1b clearly shows the deposition of $\mathrm{MAPbBr}_{3} \mathrm{NCs}$ with an average edge length of $\sim 16.8 \mathrm{~nm}$ (Fig. S2) on the surface of a $\mathrm{MoS}_{2}$ nanosheet. Fig. 1c, d are photographs of the ob- tained $\mathrm{MAPbBr}_{3} / \mathrm{MoS}_{2}$ solution under room light and UV irradiation $(365 \mathrm{~nm})$, respectively, indicating the good dispersibility and photoluminescent property of the heterostructures. Note that the average size of $\mathrm{MAPbBr}_{3} \mathrm{NCs}$ synthesized directly in solution without presence of $\mathrm{MoS}_{2}$ was $\sim 17.3 \mathrm{~nm}$ (Fig. S3) with a broader size distribution compared to those grown on $\mathrm{MoS}_{2}$ (Fig. S2). This suggests that $\mathrm{MoS}_{2}$ nanosheets as synthetic templates helped to control the nucleation process of $\mathrm{MAPbBr}_{3}$ to give a relatively narrow size distribution.

It is important to note that in our synthesis, the use of a mixed solvent to disperse $\mathrm{MoS}_{2}$ nanosheets is critical to realize the in-situ deposition of perovskite NCs on $\mathrm{MoS}_{2}$. Based on previous reports, colloidal organic-inorganic hybrid perovskite NCs are usually prepared through ligand-assisted reprecipitation (LARP) methods [38,39], which began with the dissolution of lead halide and alkylammonium halide in a polar solvent like DMF together with surfactants such as OA and OLA. This precursor solution was then injected into a nonpolar solvent (e.g., toluene or hexane) under stirring, during which perovskite NCs were precipitated due to the decrease of solubility. To in-situ deposit perovskite NCs on 2D materials like $\mathrm{MoS}_{2}$ nanosheets, the nanosheets as synthetic templates need to be dispersed in the nonpolar solvent. However, due to surface charges [42], as-exfoliated $\mathrm{MoS}_{2}$ nanosheets cannot be dispersed in a nonpolar solvent. As evidently shown in Fig. 1e, while $\mathrm{MoS}_{2}$ nanosheets were well-dispersed in DMF, they aggregated in toluene. Interestingly, when a small amount of DMF was added to toluene, for example, with a $V_{\text {DMF }}: V_{\text {toluene }}$ volume ratio of 1:30, the $\mathrm{MoS}_{2}$ nanosheets were dispersed again after sonication, and the maximum concentration could reach $\sim 0.9 \mathrm{mg} \mathrm{mL}^{-1}$ (Fig. S4). To find the optimal solvent condition for our synthesis, the critical DMF concentrations (as in toluene) at which $\mathrm{MoS}_{2}$ nanosheets began to aggregate or $\mathrm{MAPbBr}_{3}$ began to precipitate were determined, respectively (Fig. 1f). For a given $\mathrm{MoS}_{2} / \mathrm{PbBr}_{2}$ ratio (i.e., $1 \mathrm{mg} \mathrm{MoS}_{2} / 0.8 \mathrm{mmol} \mathrm{PbBr}_{2}$ ), the blue-shaded and red-shaded areas, which correspond to $\mathrm{MoS}_{2}$ aggregation and $\mathrm{MAPbBr}_{3}$ dissolution, respectively, should be avoided. The optimized condition used in the present study was presented as spot A in Fig. 1f, corresponding to a DMF concentration of $3.2 v \%$ in toluene. In such case, the presence of a small amount of DMF ensured the good dispersion of $\mathrm{MoS}_{2}$ nanosheets, and meanwhile maintained a low solvent polarity for the precipitation of perovskite NCs.

The XRD pattern of the heterostructures in Fig. $1 \mathrm{~g}$ shows the characteristic peaks for cubic-phased $(P m \overline{3} m)$ 
a
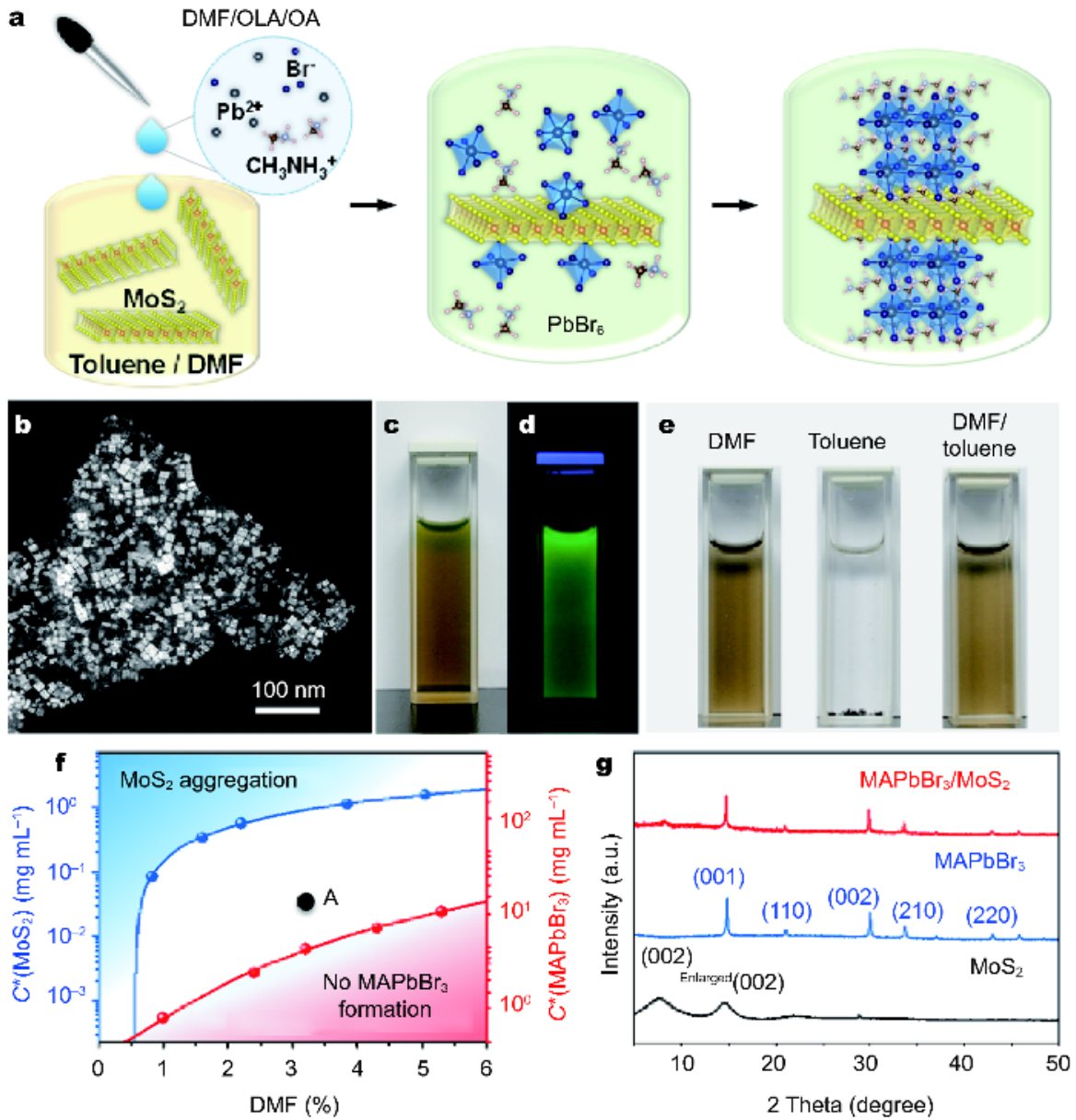

Figure 1 (a) Schematic illustration of the formation process of $\mathrm{MAPbBr}_{3} / \mathrm{MoS}_{2}$ heterostructures. (b) STEM image of a typical $\mathrm{MAPbBr}_{3} / \mathrm{MoS}_{2}$ heterostructure. Photographs of a solution containing as-synthesized $\mathrm{MAPbBr}_{3} / \mathrm{MoS}_{2}$ heterostructures (c) under room light and (d) under excitation of a $365 \mathrm{~nm}$ laser. (e) Photographs of $\mathrm{MoS}_{2}$ nanosheets in toluene, DMF and toluene/DMF, respectively. (f) Plots of critical concentrations at which (blue) $\mathrm{MoS}_{2}$ nanosheets began to aggregate and (red) $\mathrm{MAPbBr}_{3}$ began to precipitate in a mixed DMF/toluene solution at different DMF concentrations (0-6 v\%). (g) XRD patterns of as-prepared $\mathrm{MAPbBr}_{3} / \mathrm{MoS}_{2}$ heterostructures, $\mathrm{MAPbBr}_{3} \mathrm{NCs}$ and $\mathrm{MoS}_{2}$ nanosheets.

$\mathrm{MAPbBr}_{3}$ with $a=5.96 \AA[43,44]$. The peak at around $8^{\circ}$ can be attributed to the enlarged lattice spacing of the $\mathrm{MoS}_{2}$ (002) planes caused by lithium-intercalation (Fig. S5). The presence of the XRD peaks for both $\mathrm{MAPbBr}_{3}$ and $\mathrm{MoS}_{2}$ indicates the successful preparation of $\mathrm{MAPbBr} / \mathrm{MoS}_{2}$ heterostructures. Based on previous reports, $\mathrm{MoS}_{2}$ nanosheets prepared by lithium intercalation and exfoliation are usually composed of both the hexagonal $2 \mathrm{H}$ and trigonal $1 \mathrm{~T}$ polymorphs [45]. This was confirmed with XPS analysis (Fig. S6), based on which the concentration of the $1 \mathrm{~T}$ phase was estimated to be $\sim 84 \%$, indicating a highly metallic nature of the nanosheets $[46,47]$. HRTEM was then used to further study the microstructure of the heterostructures. As shown in Fig. 2a, an overlapping of lattice patterns of $\mathrm{MAPbBr}_{3}$ and $\mathrm{MoS}_{2}$ was observed, and the corresponding fast Fourier transformation (FFT)-generated diffraction pattern shows two sets of spots with a square symmetry (i.e., the vertices of the yellow square) and a hexgaonal symmetry (i.e., the vertices of the blue hexagon), respectively (Fig. 2b). By selecting the respective set of spots, the $\mathrm{MoS}_{2}$ [001]-zone lattice pattern and the $\mathrm{MAPbBr}_{3}[001]$-zone pattern were re-generated and shown in Fig. $2 \mathrm{c}$ and d, respectively. Along the $\mathrm{MAPbBr}_{3}[010] / \mathrm{MoS}_{2}$ [120] direction, the $\mathrm{MAPbBr}_{3}(020)$ planes are parallel with the $\mathrm{MoS}_{2}$ (010) planes with a $7 \%$ mismatch. Along the other direction, i.e., the $\mathrm{MAPbBr}_{3}[100] / \mathrm{MoS}_{2}$ [100] direction, the mismatch between $\mathrm{MAPbBr}_{3}(200)$ and $\mathrm{MoS}_{2}(2 \overline{1} 0)$ planes is as large as $47 \%$. To justify the epitaxial alignment in this direction, the domain-matching epitaxy was applied [48], 

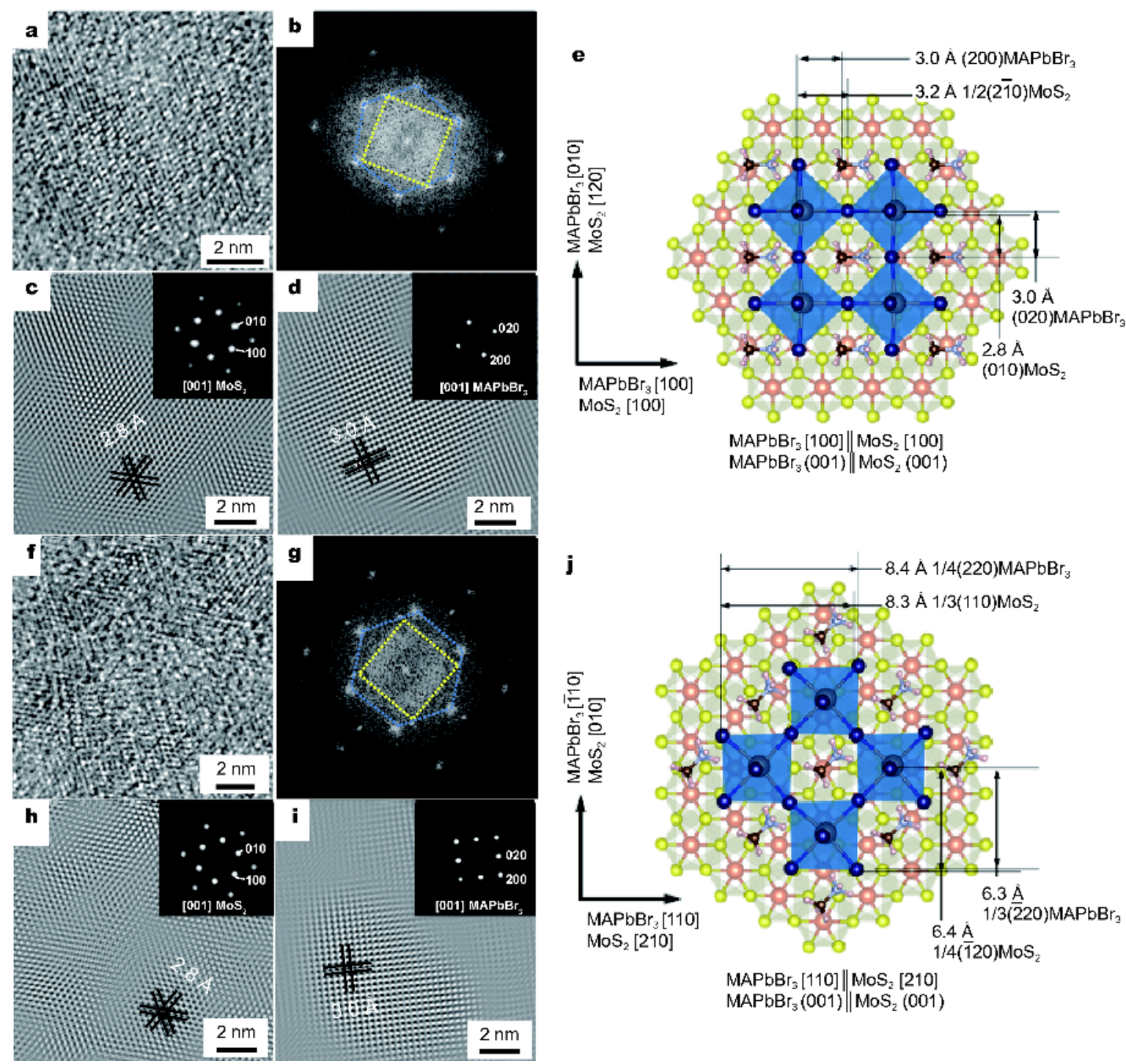

Figure 2 (a) HRTEM image of an area with overlapped lattices of $\mathrm{MAPbBr}_{3}$ and $\mathrm{MoS}_{2}$, and (b) the corresponding FFT-diffraction pattern. (c) MoS [001]-zone and (d) $\mathrm{MAPbBr}_{3}$ [001]-zone lattice patterns generated by performing inverse-FFT of the spots forming the blue hexagon and the yellow square in (b), respectively. Insets: the respectively selected spots. (e) Schematic model indicating the $\mathrm{MAPbBr}_{3}[100] \| \mathrm{MoS}_{2}[100]$ and $\mathrm{MAPbBr} \mathrm{B}_{3}(001) \|$ $\mathrm{MoS}_{2}$ (001) epitaxial relationship. (f) HRTEM image of another area with overlapped lattices of $\mathrm{MAPbBr}_{3}$ and $\mathrm{MoS}_{2}$, and (g) the corresponding FFTdiffraction pattern. (h) $\mathrm{MoS}_{2}$ [001]-zone and (i) $\mathrm{MAPbBr}_{3}$ [001]-zone lattice patterns generated by performing inverse-FFT of the spots forming the blue hexagon and the yellow square in (g), respectively. Insets: the respectively selected spots. (j) Schematic model indicating the $\mathrm{MAPbBr}_{3}[110] \|$ $\mathrm{MoS}_{2}[210]$ and $\mathrm{MAPbBr}_{3}(001) \| \mathrm{MoS}_{2}$ (001) epitaxial relationship.

in which the lattices of the two materials match through a domain that contains integral numbers of parallel atomic layers (Fig. S7). In our case, if we consider two times of the interlayer spacing of $\mathrm{MoS}_{2}$ (21̄0) planes (i.e., the spacing for $\mathrm{MoS}_{2} 1 / 2(2 \overline{1} 0)$ planes), the mismatch between it and $\mathrm{MAPbBr}_{3}(200)$ becomes only $6 \%$, as shown in the epitaxial model in Fig. 2e. Because of the different lattice symmetry between the $\mathrm{MAPbBr}_{3}(001)$ overlyer and $\mathrm{MoS}_{2}$
(001) substrate, another possible surface alignment of $\mathrm{MAPbBr}_{3}$ on $\mathrm{MoS}_{2}$ was also observed (Fig. 2f-j). The HRTEM image of another area of overlapped lattice patterns is shown in Fig. $2 \mathrm{f}$ and its FFT-generated diffraction pattern is shown in Fig. 2g, based on which lattice patterns of the $\mathrm{MAPbBr}_{3}$ [001]-zone axis and $\mathrm{MoS}_{2}$ [001]-zone axis were re-generated and shown in Fig. $2 \mathrm{~h}$, i, respectively. It can be seen that $\mathrm{MAPbBr}_{3}(220)$ and $\mathrm{MoS}_{2}$ 
(100) planes are epitaxially aligned, but with a mismatch of as large as $24 \%$. Therefore, domain-matching epitaxy is applied again. As shown in the schematic epitaxial model in Fig. 2j, the mismatch between $\mathrm{MAPbBr}_{3} 1 / 4(220)$ and $\mathrm{MoS}_{2} 1 / 3(100)$ planes along the $\mathrm{MAPbBr}_{3}[110] / \mathrm{MoS}_{2}$ [210] direction, and that between $\mathrm{MAPbBr}_{3} 1 / 3(\overline{2} 20)$ and $\mathrm{MoS}_{2} 1 / 4(\overline{1} 20)$ planes along the $\mathrm{MAPbBr}_{3}[\overline{1} 10] / \mathrm{MoS}_{2}$ [010] direction are both $\sim 1 \%$. This suggests that the epitaxial growth of $\mathrm{MAPbBr}_{3}$ on $\mathrm{MoS}_{2}$ might be structurally favored. Up to now, epitaxial heterostructures of perovskites/2D materials have only been reported recently by the physical vapor deposition (PVD) of $\mathrm{PbI}_{2}$ on a 2D material (e.g., $\mathrm{BN}$ or $\mathrm{MoS}_{2}$ ) followed by reacting $\mathrm{PbI}_{2}$ with $\mathrm{CH}_{3} \mathrm{NH}_{3} \mathrm{I}$ via $\mathrm{CVD}$ [32]. In that case, both $\mathrm{PbI}_{2}$ and the $2 \mathrm{D}$ material exhibited the six-fold symmetry at the heterointerface. It has been known that to realize epitaxial heterostructures, a small misfit in both lattice symmetry and lattice parameters is usually required to achieve a minimum interfacial energy. Some exceptions have also been reported especially in recent cases of using van der Waals layered materials as substrates which are free from surface dangling bonds to effectively tolerate strains arising from a large symmetry/lattice mismatch [49-53]. Examples include recent demonstrations of epitaxial overgrowth of $\mathrm{Pt}(101)$ on $\mathrm{MoS}_{2}(001)$ [49] and $\mathrm{PbSe}(001)$ on $\mathrm{Bi}_{2} \mathrm{Se}_{3}(001)$ [50]. Similarly, in our present work, the epitaxial deposition of $\mathrm{MAPbBr}_{3}$ on $\mathrm{MoS}_{2}$ with four-fold and six-fold symmetry at the interface, repectively, was realized in solution. This can be due to the dangling-bond-free surfaces of $\mathrm{MoS}_{2}$ nanosheets, and their soft/flexible nature when dispersed in solution to tolerate the large symmetry mismatch [50]. For comparison, we carried out a control experiment by directly mixing pre-synthesized $\mathrm{MAPbBr}_{3} \mathrm{NCs}$ and $\mathrm{MoS}_{2}$ nanosheets. $\mathrm{MAPbBr}_{3} \mathrm{NCs}$ were mostly separated from the $\mathrm{MoS}_{2}$ nanosheets without contact, and epitaxial relationship was not observed (Fig. S8).

UV-vis absorption and PL spectra of the $\mathrm{MAPbBr}_{3} /$ $\mathrm{MoS}_{2}$ heterostructure in comparison with those of $\mathrm{MAPbBr}_{3}$ NCs and $\mathrm{MoS}_{2}$ nanosheets without hybridization are shown in Fig. 3. The $\mathrm{MAPbBr}_{3} \mathrm{NCs}$ show an absorption peak at $\sim 525 \mathrm{~nm}$, corresponding to its direct band gap of $2.3 \mathrm{eV}$ [28]. Compared to $\mathrm{MAPbBr}_{3}$ or $\mathrm{MoS}_{2}$, the heterostructure exhibited increased absorption in the UV-vis region. As for the PL property, the emission of $\mathrm{MAPbBr}_{3} \mathrm{NCs}$ at $\sim 530 \mathrm{~nm}$ was dramatically quenched (by more than 90\%) when they were in-situ deposited on $\mathrm{MoS}_{2}$ nanosheets, based on the equal $\mathrm{Pb}^{2+}$ concentration (Fig. 3b). The $\mathrm{MoS}_{2}$ nanosheets exhibited no visible PL
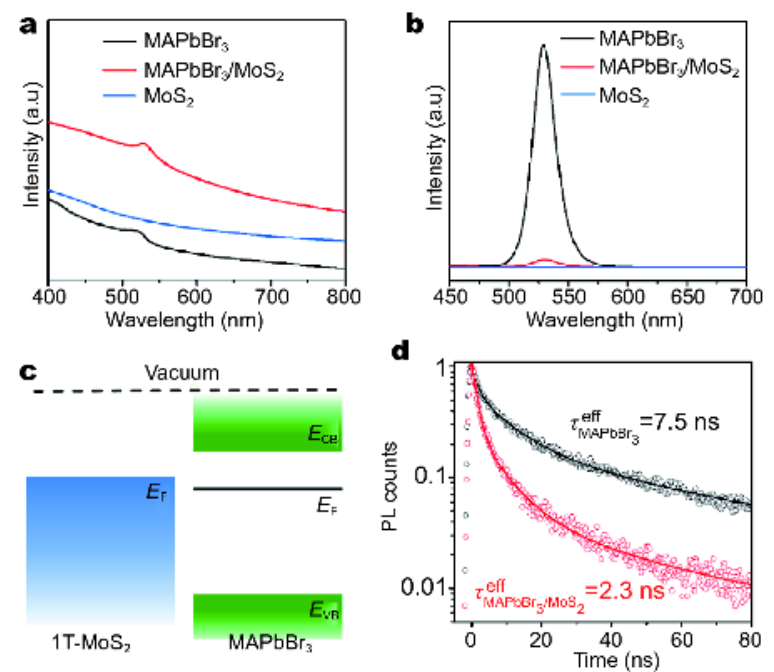

Figure 3 (a) UV-vis absorption and (b) PL spectra of the MAPbBr NCs, $\mathrm{MoS}_{2}$ nanosheets and $\mathrm{MAPbBr}_{3} / \mathrm{MoS}_{2}$ heterostructures. (c) Schematic illustration of the energy band alignment at the $\mathrm{MAPbBr}_{3} / \mathrm{MoS}_{2}$ heterointerface. (d) TRPL spectra for $\mathrm{MAPbBr}_{3} \mathrm{NCs}$ and $\mathrm{MAPbBr}_{3} / \mathrm{MoS}_{2}$ heterostructures.

emission due to a high concentration of metallic $1 \mathrm{~T}$ phase. The largely reduced PL intensity of $\mathrm{MAPbBr}_{3}$ after hybridization with $\mathrm{MoS}_{2}$ (Fig. 3b) suggests the effective energy transfer between them. This was also observed previously in perovskite/MoS $\mathrm{M}_{2}$ heterojunctions prepared by layer-by-layer assembly [36,37]. Indeed, the Fermi level of $1 \mathrm{~T} \mathrm{MoS}_{2}(\sim 3.9 \mathrm{eV})$ [45] was located below the conductive band of $\mathrm{MAPbBr}_{3}(\sim 3.6 \mathrm{eV})$ [54] as schematically shown in Fig. 3c, which might enable the effective transport of charge carriers and excitons from $\mathrm{MAPbBr}_{3}$ to $\mathrm{MoS}_{2}$. The efficient energy transfer between them was further verified by TRPL. As shown in Fig. 3d, TRPL spectra were collected at the emission center of $\mathrm{MAPbBr}_{3}$ (i.e., $530 \mathrm{~nm}$ ). The effective decay lifetime of the pure $\mathrm{MAPbBr}_{3}\left(\tau_{\mathrm{MAPBr}_{3}}^{\text {eff }}\right)$ was measured as $7.5 \mathrm{~ns}$ while that for $\mathrm{MAPbBr}_{3} / \mathrm{MoS}_{2}\left(\tau^{\text {eff }} \mathrm{MAPBBr}_{3} / \mathrm{Mos}_{2}\right)$ was $2.3 \mathrm{~ns}$. This difference is likely caused by the energy transfer from $\mathrm{MAPbBr}_{3}$ to $\mathrm{MoS}_{2}$, and the transfer time, $\tau_{\mathrm{ET}}$, as estimated based on the equation below [21] is $3.3 \mathrm{~ns}$, which is in the ns time scale of radiative recombination that ensures efficient PL quenching [21,55].

$$
\frac{1}{\tau_{\mathrm{MAPBBr}_{3} \mathrm{MoS}_{2}}^{\text {eff }}}=\frac{1}{\tau_{\mathrm{MAPBBr}_{3}}^{e \text { eff }}}+\frac{1}{\tau_{\mathrm{ET}}}
$$

The solution-processible $\mathrm{MAPbBr}_{3} / \mathrm{MoS}_{2}$ heterostructures prepared from the facile and scalable approach are suitable for fabrication of thin-film devices. As a proof-of-concept demonstration, paper-based photo- 
a
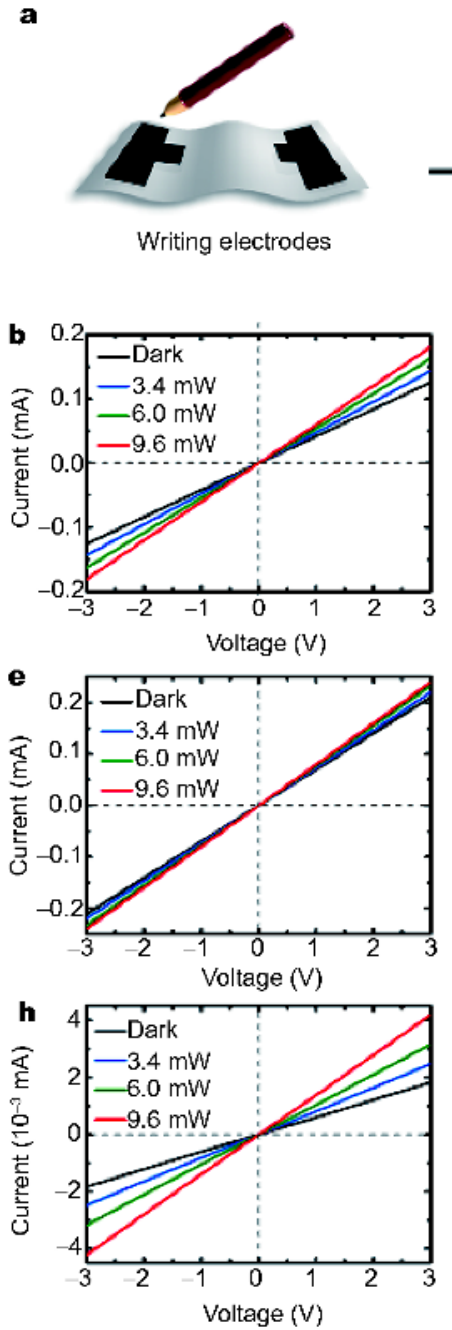

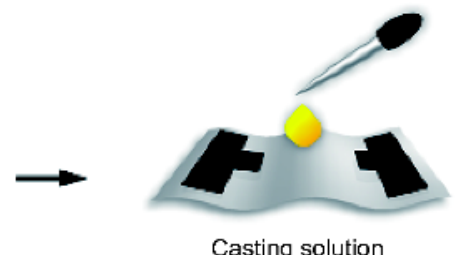

Casting solution
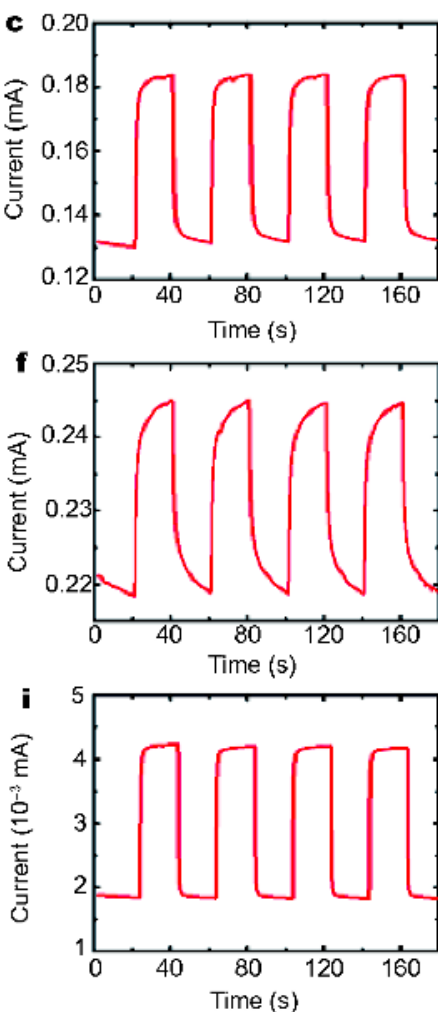
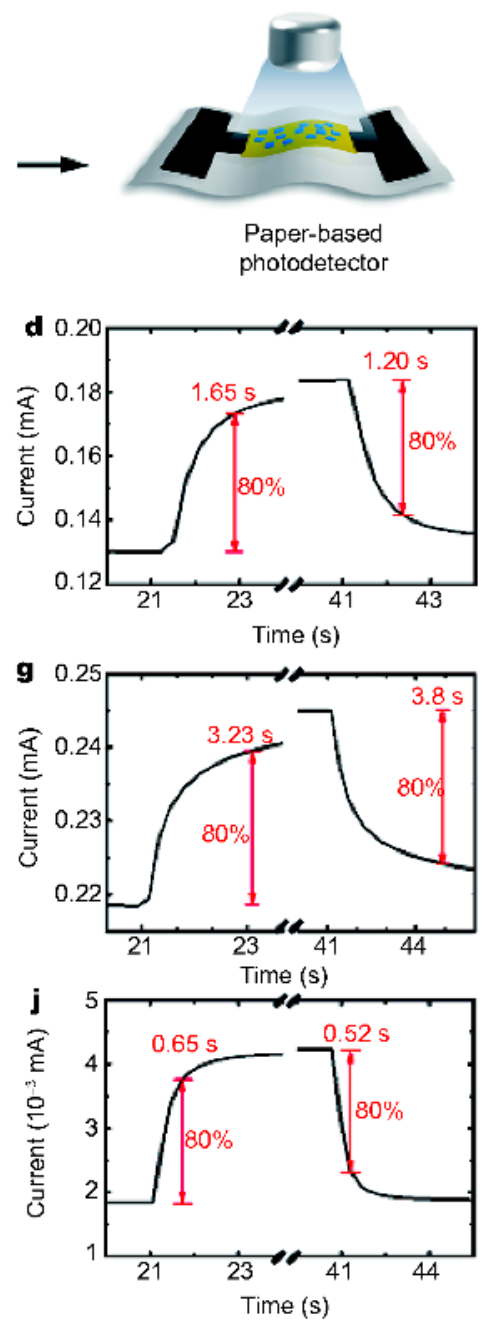

Figure 4 (a) Schematic illustration of the fabrication process of a paper-based photodetector. (b) $I-V$ curves at different light intensity, (c) temporal photocurrent response and (d) a zoom-in view of the temporal photocurrent response of the photodetector based on $\mathrm{MAPbBr}_{3} / \mathrm{MoS}_{2}$ heterostructures. (e) $I-V$ curves, (f) temporal photocurrent response and (g) a zoom-in view of the temporal photocurrent response of the photodetector based on $\mathrm{MoS}_{2}$ nanosheets. (h) $I-V$ curves, (i) temporal photocurrent response and (j) a zoom-in view of the temporal photocurrent response of the photodetector based on $\mathrm{MAPbBr}_{3} / \mathrm{MoS}_{2}$ heterostructures after they were partially transformed from $1 \mathrm{~T}$ to $2 \mathrm{H}$ phase by irradiation with a $780 \mathrm{~nm}$ laser. The light source used for all measurements was a $405 \mathrm{~nm}$ laser.

detectors were fabricated based on the $\mathrm{MAPbBr}_{3} / \mathrm{MoS}_{2}$ heterostructures. Compared to the conventional silicon substrate and plastic substrates such as polyethylene terephthalate (PET) and polydimethylsiloxane (PDMS), paper is cheap and renewable and has been explored as a supplement to silicon and plastics in many areas [56,57]. More importantly, pencil traces (graphite) could be easily drawn on paper as electrodes, and the whole fabrication process takes $\sim 10 \mathrm{~min}$, much faster than making devices on plastics or silicon which normally requires thermally evaporated metal electrodes through a shadow mask
$[34,35]$. As schematically shown in Fig. 4a, two graphite electrodes were firstly drawn on a piece of paper by using a 12B pencil (Fig. S9). After that, a solution of the heterostructures was drop-casted between the electrodes and then dried naturally before measurements. The $\mathrm{MAPbBr}_{3} / \mathrm{MoS}_{2}$ heterostructures deposited on paper formed a continuous film without obvious cracks (Fig. $\mathrm{S} 10 \mathrm{a}-\mathrm{c})$. The linear and symmetrical current-voltage $(I-V)$ curve of the device suggests that the $\mathrm{MAPbBr}_{3} /$ $\mathrm{MoS}_{2}$ heterostructures formed a low resistance contact with the pencil trace electrodes (Fig. S10d) [58]. Fig. 4b 
shows the $I-V$ curves of the $\mathrm{MAPbBr}_{3} / \mathrm{MoS}_{2}$ paper-based photodetector under $405 \mathrm{~nm}$ laser illumination at varied power from 3.4 to $9.6 \mathrm{~mW}$. A clear rise of the photocurrent with increasing illumination intensity was observed, indicating the effective conversion of photon flux to photogenerated carriers. However, the observed low on/off ratio, with a value of 1.41 at $9.6 \mathrm{~mW}$, was due to the relatively large dark current which was caused by the high concentration of metallic $1 \mathrm{~T}$ phase in the $\mathrm{MoS}_{2}$ nanosheets [37]. The temporal photoresponse of the photodetector was measured under the periodic illumination of a $405 \mathrm{~nm}$ laser with an on/off interval of $20 \mathrm{~s}$ at a bias of $3 \mathrm{~V}$. The $\mathrm{MAPbBr}_{3} / \mathrm{MoS}_{2}$ paper-based photodetector showed good on-off switching as shown in Fig. 4c. The rise time and decay time, as also important parameters for evaluating a photodetector, were measured as the time required for the photoresponse to increase by $80 \%$ from the minimum current and drop by $80 \%$ from the maximum current, respectively [37]. As shown in Fig. 4d, the rise and fall time of the $\mathrm{MAPbBr}_{3} /$ $\mathrm{MoS}_{2}$ hybrid device was 1.65 and $1.20 \mathrm{~s}$, respectively. Compared to the $\mathrm{MoS}_{2}$-based device (Fig. 4e-g), the device based on $\mathrm{MAPbBr}{ }_{3} / \mathrm{MoS}_{2}$ showed the improved on/ off ratio, photoresponsivity and response rate (Fig. $4 \mathrm{~b}-\mathrm{g}$ and Table S1). Such improvement can be attributed to the increased absorption of $\mathrm{MAPbBr}_{3} / \mathrm{MoS}_{2}$ heterostructures compared to the pure $\mathrm{MoS}_{2}$ (Fig. 3a), the effective energy transfer from $\mathrm{MAPbBr}_{3}$ to $\mathrm{MoS}_{2}$ (Fig. 3d), and a possibly higher carrier mobility within $\mathrm{MAPbBr}_{3} \mathrm{NCs}$ compared to $\mathrm{MoS}_{2}[59,60]$. In addition, a device based on $\mathrm{MAPbBr}_{3}$ NCs showed an extremely low photoresponsivity of $\sim 7 \times 10^{-8} \mathrm{~mA} \mathrm{~W}^{-1}$ (Fig. S11). This could be attributed to their poor film-forming ability when deposited on a piece of paper (Fig. S12), which is in sharp contrast to the crack-free film formed by $\mathrm{MAPbBr}_{3} / \mathrm{MoS}_{2}$ heterostructures (Fig. S10). Based on the aforementioned TRPL analysis in Fig. 3d, it can be expected that under laser illumination, charge carriers and/or excitons were generated in the $\mathrm{MAPbBr}_{3} \mathrm{NCs}$ and readily transferred to the nearby $\mathrm{MoS}_{2}$ nanosheets, leading to the shortened PL lifetime of $\mathrm{MAPbBr}_{3} / \mathrm{MoS}_{2}$ compared to $\mathrm{MAPbBr}_{3}$ alone. The charge carriers and excitons were then separated in $\mathrm{MoS}_{2}$ under the applied electric field, which finally contributed to the photocurrent. The presence of $\mathrm{MAPbBr}_{3}$ NCs, despite of their poor film-forming ability, therefore compensated the poor light adsorption and photo-conversion ability of $\mathrm{MoS}_{2}$ nanosheets with a high metallic phase concentration. Evidently, the photoresponsivity of the $\mathrm{MAPbBr}_{3} / \mathrm{MoS}_{2}$ device $\left(5.6 \mathrm{~mA} \mathrm{~W}{ }^{-1}\right)$ doubled compared to that of $\mathrm{MoS}_{2}\left(2.76 \mathrm{~mA} \mathrm{~W}{ }^{-1}\right)$ and increased for at least $10^{7}$ times compared with $\mathrm{MAPbBr}_{3} \mathrm{NCs}\left(\sim 7 \times 10^{-8}\right.$ $\mathrm{mA} \mathrm{W}^{-1}$ ) alone. Moreover, we further compared performances of $\mathrm{MAPbBr}_{3} / \mathrm{MoS}_{2}$ epitaxial heterostructures with physically mixed $\mathrm{MAPbBr}_{3} / \mathrm{MoS}_{2}$ composite by depositing them on paper as photodetectors, and found that the in-situ prepared heterostructures exhibited higher photoresponsivity and on/off ratio compared with the physically mixed counterpart (Fig. 4, Fig. S13, and Table S1). Above observations further indicate the importance of using $\mathrm{MoS}_{2}$ nanosheets as flexible substrates that connected dispersed perovskite NCs, and also enabled the effective charge and/or exciton transport through the epitaxial interfaces. The stability of the $\mathrm{MAPbBr}_{3} / \mathrm{MoS}_{2}-$ based device was also investigated (Fig. S14), which exhibited stable current density for 2,000 s under temporal illumination by a $405 \mathrm{~nm}$ laser with a power of $9.6 \mathrm{~mW}$, suggesting that degradation of the perovskite or phase transition in $\mathrm{MoS}_{2}$ did not happen during the test. This is also confirmed by comparing the XRD patterns of the $\mathrm{MAPbBr}{ }_{3} / \mathrm{MoS}_{2}$ hererostructures before and after $30 \mathrm{~min}$ of $405 \mathrm{~nm}$ laser irradiation, and no additional peaks due to degradation were observed (Fig. S15).

The electronic property of $\mathrm{MoS}_{2}$ is highly dependent on its crystal structure. While $2 \mathrm{H}$-phased $\mathrm{MoS}_{2}$ is semiconducting, 1T-phased counterpart is metal-like [46]. The Li-intercalated and exfoliated $\mathrm{MoS}_{2}$ nanosheets contain $\sim 84 \%$ of $1 \mathrm{~T}$ phase (Fig. S6 in SI), indicating a highly metallic nature. As a result, photodetector based on the metallic $\mathrm{MoS}_{2}$ nanosheets hybridized with $\mathrm{MAPbBr}_{3} \mathrm{NCs}$ showed a relatively high dark current density, leading to a relatively low on/off ratio of 1.4. It has been reported previously that metallic $1 \mathrm{~T} \mathrm{MoS}_{2}$ can be reverted back to the semiconducting $2 \mathrm{H}$ phase by annealing [46] or laser irradiation [61]. We therefore partially converted the $\mathrm{MoS}_{2}$ from $1 \mathrm{~T}$ to $2 \mathrm{H}$ in the $\mathrm{MAPbBr} / \mathrm{MoS}_{2}$ device by simply illuminating it with a $780 \mathrm{~nm}$ laser at $100 \mathrm{~mW}$ for $30 \mathrm{~s}$, and the $2 \mathrm{H}$ concentration increased from $16 \%$ to $68 \%$ (Fig. S16), during which the $\mathrm{MAPbBr}_{3}$ NCs remained stable (Fig. S17). As expected, due to the increased channel resistance after metal-to-semiconductor transition, both dark current and photocurrent considerably decreased, leading to a decreased responsivity of $0.224 \mathrm{~mA} \mathrm{~W}^{-1}$ (Fig. 4i). Meanwhile, the on/off ratio of the $\mathrm{MAPbBr}_{3} / \mathrm{MoS}_{2}$ device after laser illumination was evidently increased from 1.41 to 2.30 (Fig. 4h). Importantly, after 1T-to-2H conversion, the response times were much shortened $(0.65$ and $0.52 \mathrm{~s})$ (Fig. 4j), which could result from the reduced defect density in $\mathrm{MoS}_{2}$ after its transition to a more thermodynamically stable structure [37]. 


\section{CONCLUSIONS}

In summary, $\mathrm{MAPbBr}_{3} \mathrm{NCs}$ were directly deposited on $\mathrm{MoS}_{2}$ in a mixed DMF/toluene solution. An optimal concentration of DMF in toluene enabled the dispersion of $\mathrm{MoS}_{2}$ nanosheets and meanwhile ensured the precipitation of $\mathrm{MAPbBr}_{3} \mathrm{NCs}$. The in-situ deposited (001)oriented $\mathrm{MAPbBr}_{3} \mathrm{NCs}$ showed epitaxial relationship with (001) $\mathrm{MoS}_{2}$ nanosheets by aligning along either the $\mathrm{MAPbBr}_{3}[100] / \mathrm{MoS}_{2}$ [100] direction or the $\mathrm{MAPbBr}_{3}$ [110]/MoS 2 [210] direction. The dispersible $\mathrm{MAPbBr}_{3} /$ $\mathrm{MoS}_{2}$ epitaxial heterostructures can be directly dropcasted between two graphite electrodes drawn by pencil on a piece of paper to form a photodetector with simple configuration, and demonstrated the much improved performance compared to using $\mathrm{MoS}_{2}$ or $\mathrm{MAPbBr}_{3}$ alone due to the improved light adsorption and enhanced energy transfer. Our work suggests that despite the stringent solvation conditions, organic-inorganic hybrid perovskites could be hybridized with other functional nanostructures via solution-phase epitaxy. 2D materials provide flexible substrates for the epitaxial deposition of crystals even with large mismatch in lattice symmetry and parameters. Thus-obtained solution-processible heterostructures are promising for thin-film optoelectronics.

\section{Received 4 April 2018; accepted 8 April 2018;} published online 11 May 2018

1 Chhowalla M, Shin HS, Eda G, et al. The chemistry of two-dimensional layered transition metal dichalcogenide nanosheets. Nat Chem, 2013, 5: 263-275

2 Huang X, Zeng Z, Zhang H. Metal dichalcogenide nanosheets: preparation, properties and applications. Chem Soc Rev, 2013, 42: 1934-1946

3 Geim AK, Novoselov KS. The rise of graphene. Nat Mater, 2007, 6: 183-191

4 Karunadasa HI, Montalvo E, Sun Y, et al. A molecular $\mathrm{MoS}_{2}$ edge site mimic for catalytic hydrogen generation. Science, 2012, 335: 698-702

$5 \mathrm{Yu}$ WJ, Li Z, Zhou $\mathrm{H}$, et al. Vertically stacked multi-heterostructures of layered materials for logic transistors and complementary inverters. Nat Mater, 2013, 12: 246-252

6 Yu WJ, Liu Y, Zhou H, et al. Highly efficient gate-tunable photocurrent generation in vertical heterostructures of layered materials. Nat Nanotechnol, 2013, 8: 952-958

7 Amani M, Lien DH, Kiriya D, et al. Near-unity photoluminescence quantum yield in $\mathrm{MoS}_{2}$. Science, 2015, 350: 1065-1068

8 Eda G, Maier SA. Two-dimensional crystals: managing light for optoelectronics. ACS Nano, 2013, 7: 5660-5665

9 Zhang $\mathrm{W}$, Chuu $\mathrm{CP}$, Huang JK, et al. Ultrahigh-gain photodetectors based on atomically thin graphene- $\mathrm{MoS}_{2}$ heterostructures. Sci Rep, 2014, 4: 3826

10 Tan $\mathrm{H}, \mathrm{Xu} \mathrm{W}$, Sheng Y, et al. Lateral graphene-contacted vertically stacked $\mathrm{WS}_{2} / \mathrm{MoS}_{2}$ hybrid photodetectors with large gain. Adv Mater, 2017, 29: 1702917
11 Lin J, Li H, Zhang $\mathrm{H}$, et al. Plasmonic enhancement of photocurrent in $\mathrm{MoS}_{2}$ field-effect-transistor. Appl Phys Lett, 2013, 102: 203109

$12 \mathrm{Yu}$ SH, Lee Y, Jang SK, et al. Dye-sensitized $\mathrm{MoS}_{2}$ photodetector with enhanced spectral photoresponse. ACS Nano, 2014, 8: 82858291

13 Esmaeili-Rad MR, Salahuddin S. High performance molybdenum disulfide amorphous silicon heterojunction photodetector. Sci Rep, 2013, 3: 2345

14 Jariwala D, Sangwan VK, Wu CC, et al. Gate-tunable carbon nanotube- $\mathrm{MoS}_{2}$ heterojunction p-n diode. Proc Natl Acad Sci USA, 2013, 110: 18076-18080

15 Noel NK, Stranks SD, Abate A, et al. Lead-free organic-inorganic tin halide perovskites for photovoltaic applications. Energy Environ Sci, 2014, 7: 3061-3068

16 Jeon T, Kim SJ, Yoon J, et al. Hybrid perovskites: effective crystal growth for optoelectronic applications. Adv Energy Mater, 2017, 7: 1602596

17 Sum TC, Mathews N. Advancements in perovskite solar cells: photophysics behind the photovoltaics. Energy Environ Sci, 2014, 7: $2518-2534$

18 Yang WS, Noh JH, Jeon NJ, et al. High-performance photovoltaic perovskite layers fabricated through intramolecular exchange. Science, 2015, 348: 1234-1237

19 Chiang $\mathrm{CH}$, Nazeeruddin MK, Grätzel M, et al. The synergistic effect of $\mathrm{H}_{2} \mathrm{O}$ and DMF towards stable and $20 \%$ efficiency inverted perovskite solar cells. Energy Environ Sci, 2017, 10: 808-817

20 Saliba M, Matsui T, Domanski K, et al. Incorporation of rubidium cations into perovskite solar cells improves photovoltaic performance. Science, 2016, 354: 206-209

21 Xing G, Mathews N, Sun S, et al. Long-range balanced electronand hole-transport lengths in organic-inorganic $\mathrm{CH}_{3} \mathrm{NH}_{3} \mathrm{PbI}_{3}$. Science, 2013, 342: 344-347

22 Stranks SD, Eperon GE, Grancini G, et al. Electron-hole diffusion lengths exceeding 1 micrometer in an organometal trihalide perovskite absorber. Science, 2013, 342: 341-344

23 Green MA, Ho-Baillie A, Snaith HJ. The emergence of perovskite solar cells. Nat Photonics, 2014, 8: 506-514

24 Schmidt LC, Pertegás A, González-Carrero S, et al. Nontemplate synthesis of $\mathrm{CH}_{3} \mathrm{NH}_{3} \mathrm{PbBr}_{3}$ perovskite nanoparticles. J Am Chem Soc, 2014, 136: 850-853

25 Pathak S, Sakai N, Wisnivesky Rocca Rivarola F, et al. Perovskite crystals for tunable white light emission. Chem Mater, 2015, 27: 8066-8075

26 Gonzalez-Carrero S, Galian RE, Pérez-Prieto J. Maximizing the emissive properties of $\mathrm{CH}_{3} \mathrm{NH}_{3} \mathrm{PbBr}_{3}$ perovskite nanoparticles. J Mater Chem A, 2015, 3: 9187-9193

27 Huang $\mathrm{H}$, Zhao F, Liu L, et al. Emulsion synthesis of size-tunable $\mathrm{CH}_{3} \mathrm{NH}_{3} \mathrm{PbBr}_{3}$ quantum dots: an alternative route toward efficient light-emitting diodes. ACS Appl Mater Interfaces, 2015, 7: 2812828133

28 Zhu F, Men L, Guo Y, et al. Shape evolution and single particle luminescence of organometal halide perovskite nanocrystals. ACS Nano, 2015, 9: 2948-2959

29 Jang DM, Kim DH, Park K, et al. Ultrasound synthesis of lead halide perovskite nanocrystals. J Mater Chem C, 2016, 4: 1062510629

30 Xing J, Yan F, Zhao Y, et al. High-efficiency light-emitting diodes of organometal halide perovskite amorphous nanoparticles. ACS Nano, 2016, 10: 6623-6630 
31 Im JH, Lee CR, Lee JW, et al. 6.5\% Efficient perovskite quantumdot-sensitized solar cell. Nanoscale, 2011, 3: 4088-4093

32 Niu L, Liu X, Cong C, et al. Controlled synthesis of organic/inorganic van der Waals solid for tunable light-matter interactions. Adv Mater, 2015, 27: 7800-7808

33 Cheng HC, Wang G, Li D, et al. van der Waals heterojunction devices based on organohalide perovskites and two-dimensional materials. Nano Lett, 2015, 16: 367-373

$34 \mathrm{Lu}$ J, Carvalho A, Liu H, et al. Hybrid bilayer WSe $\mathrm{W}_{2}-\mathrm{CH}_{3} \mathrm{NH}_{3} \mathrm{PbI}_{3}$ organolead halide perovskite as a high-performance photodetector. Angew Chem Int Ed, 2016, 55: 11945-11949

35 Ma C, Shi Y, Hu W, et al. Heterostructured $\mathrm{WS}_{2} / \mathrm{CH}_{3} \mathrm{NH}_{3} \mathrm{PbI}_{3}$ photoconductors with suppressed dark current and enhanced photodetectivity. Adv Mater, 2016, 28: 3683-3689

36 Kang DH, Pae SR, Shim J, et al. An ultrahigh-performance photodetector based on a perovskite-transition-metal-dichalcogenide hybrid structure. Adv Mater, 2016, 28: 7799-7806

37 Wang Y, Fullon R, Acerce M, et al. Solution-processed $\mathrm{MoS}_{2} /$ organolead trihalide perovskite photodetectors. Adv Mater, 2017, 29: 1603995

38 Zhang F, Zhong $\mathrm{H}$, Chen $\mathrm{C}$, et al. Brightly luminescent and colortunable colloidal $\mathrm{CH}_{3} \mathrm{NH}_{3} \mathrm{PbX}_{3}(\mathrm{X}=\mathrm{Br}, \mathrm{I}, \mathrm{Cl})$ quantum dots: potential alternatives for display technology. ACS Nano, 2015, 9: 4533-4542

39 Zhang F, Huang S, Wang P, et al. Colloidal synthesis of air-stable $\mathrm{CH}_{3} \mathrm{NH}_{3} \mathrm{PbI}_{3}$ quantum dots by gaining chemical insight into the solvent effects. Chem Mater, 2017, 29: 3793-3799

40 Zeng Z, Yin Z, Huang X, et al. Single-layer semiconducting nanosheets: high-yield preparation and device fabrication. Angew Chem Int Ed, 2011, 50: 11093-11097

$41 \mathrm{Li} \mathrm{H}, \mathrm{Wu} \mathrm{J}$, Yin Z, et al. Preparation and applications of mechanically exfoliated single-layer and multilayer $\mathrm{MoS}_{2}$ and $\mathrm{WSe}_{2}$ nanosheets. Acc Chem Res, 2014, 47: 1067-1075

42 Heising J, Kanatzidis MG. Exfoliated and restacked $\mathrm{MoS}_{2}$ and $\mathrm{WS}_{2}$ : ionic or neutral species? Encapsulation and ordering of hard Electropositive cations. J Am Chem Soc, 1999, 121: 11720-11732

43 Peng W, Wang L, Murali B, et al. Solution-grown monocrystalline hybrid perovskite films for hole-transporter-free solar cells. Adv Mater, 2016, 28: 3383-3390

44 Brunetti B, Cavallo C, Ciccioli A, et al. On the thermal and thermodynamic (in)stability of methylammonium lead halide perovskites. Sci Rep, 2016, 6: 31896

45 Kappera R, Voiry D, Yalcin SE, et al. Phase-engineered low-resistance contacts for ultrathin $\mathrm{MoS}_{2}$ transistors. Nat Mater, 2014, 13: $1128-1134$

46 Eda G, Yamaguchi H, Voiry D, et al. Photoluminescence from chemically exfoliated $\mathrm{MoS}_{2}$. Nano Lett, 2011, 11: 5111-5116

47 Voiry D, Salehi M, Silva R, et al. Conducting $\mathrm{MoS}_{2}$ nanosheets as catalysts for hydrogen evolution reaction. Nano Lett, 2013, 13: 6222-6227

48 Narayan J, Larson BC. Domain epitaxy: A unified paradigm for thin film growth. J Appl Phys, 2003, 93: 278-285

49 Huang X, Zeng Z, Bao S, et al. Solution-phase epitaxial growth of noble metal nanostructures on dispersible single-layer molybdenum disulfide nanosheets. Nat Commun, 2013, 4: 1444

50 Lin Z, Yin A, Mao J, et al. Scalable solution-phase epitaxial growth of symmetry-mismatched heterostructures on two-dimensional crystal soft template. Sci Adv, 2016, 2: e1600993

51 Jin $\mathrm{M}$, Zhang H, Wang J, et al. Copper can still be epitaxially deposited on palladium nanocrystals to generate core-shell nanocubes despite their large lattice mismatch. ACS Nano, 2012, 6: 2566-2573

52 Fan FR, Liu DY, Wu YF, et al. Epitaxial growth of heterogeneous metal nanocrystals: from gold nano-octahedra to palladium and silver nanocubes. J Am Chem Soc, 2008, 130: 6949-6951

53 Geim AK, Grigorieva IV. van der Waals heterostructures. Nature, 2013, 499: 419-425

54 Schulz P, Edri E, Kirmayer S, et al. Interface energetics in organometal halide perovskite-based photovoltaic cells. Energy Environ Sci, 2014, 7: 1377-1381

55 Yang D, Yang R, Zhang J, et al. High efficiency flexible perovskite solar cells using superior low temperature $\mathrm{TiO}_{2}$. Energy Environ Sci, 2015, 8: 3208-3214

56 Fang H, Li J, Ding J, et al. An origami perovskite photodetector with spatial recognition ability. ACS Appl Mater Interfaces, 2017, 9: 10921-10928

57 Lin CH, Tsai DS, Wei TC, et al. Highly deformable origami paper photodetector arrays. ACS Nano, 2017, 11: 10230-10235

58 Cai C, Ma Y, Jeon J, et al. Epitaxial growth of large-grain NiSe films by solid-state reaction for high-responsivity photodetector arrays. Adv Mater, 2017, 29: 1606180

59 Lopez-Sanchez O, Lembke D, Kayci M, et al. Ultrasensitive photodetectors based on monolayer $\mathrm{MoS}_{2}$. Nat Nanotechnol, 2013, 8: 497-501

60 Stranks SD, Snaith HJ. Metal-halide perovskites for photovoltaic and light-emitting devices. Nat Nanotechnol, 2015, 10: 391-402

61 Fan X, Xu P, Zhou D, et al. Fast and efficient preparation of exfoliated $2 \mathrm{H} \mathrm{MoS}_{2}$ nanosheets by sonication-assisted lithium intercalation and infrared laser-induced $1 \mathrm{~T}$ to $2 \mathrm{H}$ phase reversion. Nano Lett, 2015, 15: 5956-5960

Acknowledgements This research was supported by the National Natural Science Foundation of China (51322202), and the Young 1000 Talents Global Recruitment Program of China. Xing G acknowledges the financial support from Macau Science and Technology Development Fund (FDCT-116/2016/A3 and FDCT-091/2017/A2), Research Grant (SRG2016-00087-FST) from the University of Macau, the Natural Science Foundation of China (91733302, 61605073 and 2015CB932200), and the Young 1000 Talents Global Recruitment Program of China.

Author contributions Huang X, Xing G, and Huang W conceived the idea. Zhang Z, Sun $F$ designed the experiments and synthesized the hybrid nanomaterial. Dai J and Sun Q exfoliated $\mathrm{MoS}_{2}$ nanosheets. Zhu $\mathrm{Z}$, Gao $\mathrm{K}$ and Shi X performed TEM, XRD and AFM measurements. Wei Q conducted PL and TRPL measurements. Li H, Yan Y and Yu H designed the paper-based photodetectors. All authors contributed to the general discussion.

Conflict of interest The authors declare no conflict of interest.

Supplementary information Experimental details and supporting data are available in the online version of the paper. 

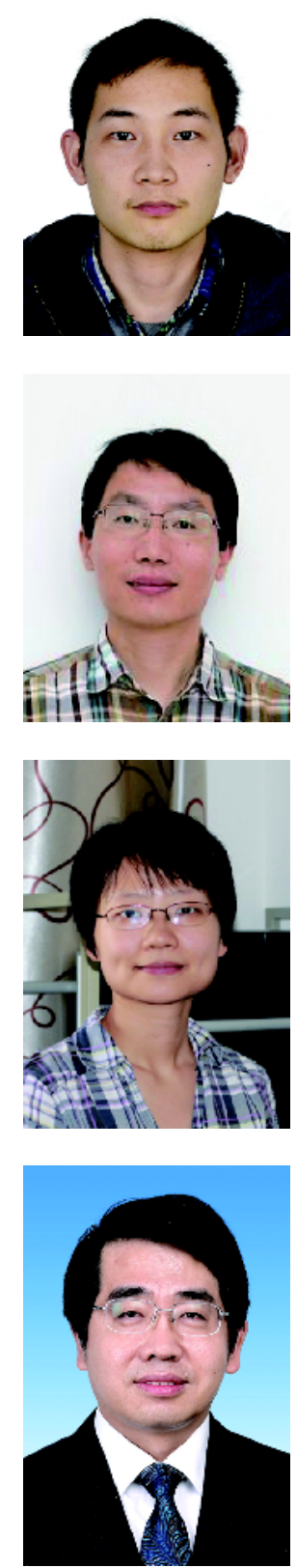

Zhipeng Zhang received his bachelor degree in 2015 at Nanjing Tech University. He is now a master student in the Institute of Advanced Materials (IAM) of Nanjing Tech University. His current research interest is the synthesis and applications of perovskite nanocrystals.
Guichuan Xing obtained his BSc in 2003 at Fudan University and PhD in 2011 at National University of Singapore. He joined IAM of Nanjing Tech University as a professor in 2014. His current research interest is nonlinear optical properties and ultrafast carrier dynamics in novel optoelectronic materials and devices.
Xiao Huang received her bachelor's degree from the School of Materials Science and Engineering at Nanyang Technological University in Singapore in 2006 and completed her PhD in 2011 under the supervision of Prof. Hua Zhang and Prof. Freddy Boey. She is currently a professor at the Institute of Advanced Materials (IAM), Nanjing Tech University. Her research interest includes the synthesis and applications of two-dimensional nanomaterial-based hybrids.

\section{非传统溶液外延法在金属硫化物纳米片表面生长有机无机杂化钻钠矿纳米晶}

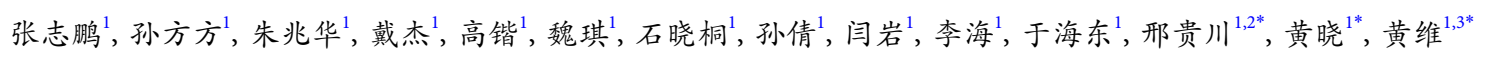

摘要 基于外延异质结构的有机-无机杂化钲钛矿/二维纳米片复合材料在光电领域具有很好的应用前景, 但目前使用的固相制备方法大 大限制了这一目标的实现. 我们通过精细调节溶剂环境, 成功利用外延沉积的方式实现了在三角/六方相 $M_{0} \mathrm{~S}_{2}$ 纳米片表面生长立方相

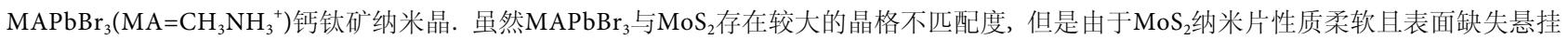
键, 可以在两条不同方向上观察到较高容忍度 ( 1\%错位)的外延生长关系. 这种外延界面的形成有利于 $\mathrm{MAPbBr}_{3}$ 与 $\mathrm{MoS}_{2}$ 之间有效的能量转 移, 因此基于 $\mathrm{MAPbBr}_{3} / \mathrm{MoS}_{2}$ 异质结的纸质器件与 $\mathrm{MAPbBr}_{3}$ 或 $\mathrm{MoS}_{2}$ 器件相比具有更优异的光电性能. 此外, 除了提高光吸收能力和能量传 递, $\mathrm{MoS}_{2}$ 纳米片的存在还为离散的 $\mathrm{MAPbBr}_{3}$ 纳米晶提供柔性和连续的基底, 从而改善了 $\mathrm{MAPbBr}_{3}$ 纳米晶粒的成膜能力. 这种液相外延法可 用于高性能的有机无机杂化钻钛矿与二维材料的异质结构材料的大规模制备, 将推动异质结构材料在光电领域的广泛使用. 